\title{
INFLUÊNCIA DA TEMPERATURA NA EXTRAÇÃO DE COMPOSTOS ANTIMICROBIANOS OBTIDOS DE FUNGOS COMESTÍVEIS
}

Camila Ramão Contessa ${ }^{1}$, Nathieli Bastos de Souza ${ }^{2}$, Guilherme Battú Gonçalo ${ }^{3}$, Luciano dos Santos Almeida ${ }^{4}$, Ana Paula Manera ${ }^{5}$, Caroline Costa Moraes ${ }^{6}$

${ }^{1}$ Universidade Federal do Pampa, Curso de Engenharia de Alimentos, Graduanda em Engenharia de Alimentos

${ }^{2}$ Universidade Federal do Rio Grande, Escola de Química e Alimentos, Graduada em Engenharia de Alimentos

${ }^{3}$ Universidade Federal do Pampa, Curso de Engenharia de Alimentos, Graduando em Engenharia de Alimentos

${ }^{4}$ Universidade Federal do Pampa, Curso de Engenharia de Alimentos, Graduado em Biologia

${ }^{5}$ Universidade Federal do Pampa, Curso de Engenharia de Alimentos, Doutora em Engenharia de Alimentos

${ }^{6}$ Universidade Federal do Pampa, Curso de Engenharia de Alimentos, Doutora em Engenharia de Alimentos

E-mail para contato: camilaramao@hotmail.com

RESUMO - Em decorrência da necessidade de produtos industrializados, se torna imprescindível a redução de micro-organismos deteriorantes e patógenos, a fim de se obter um alimento seguro e de qualidade. Com a grande utilização de aditivos químicos e a potencialidade de efeitos tóxicos destes produtos ao consumidor, teve-se o aumento do interesse por produtos naturais. A extração de conservantes naturais pode ser obtido a partir de fungos comestíveis, uma vez que, apresentam potencialidade terapêutica e antimicrobiana consideráveis. Neste trabalho, realizou-se a extração em diferentes temperaturas de compostos antimicrobianos do micélio de cogumelos comestíveis Pleurotus sajor-caju e Morchella esculenta, e a avaliação da sua ação sob os micro-organismos patógenos Staphylococcus aureus e Escherichia coli. Para a avaliação antimicrobiana foram feitas análises a partir da metodologia de microplacas. Os resultados sugerem que os dois gêneros de cogumelos possuem ação antimicrobiana sob os patógenos estudados e que a temperatura interfere na extração destes compostos: para o gênero Pleurotus, a temperatura de $60^{\circ} \mathrm{C}$ apresentou um maior resultado contra a Escherichia coli e a temperatura de $25^{\circ} \mathrm{C}$ se mostrou mais eficaz sob Staphylococcus aureus. Constata-se ainda que as diferentes temperaturas de extração testadas promoveram uma extração semelhante de compostos antibacterianos frente a microbiota utilizada. Evidenciando que a extração feita do micélio do fungo a temperatura de $25^{\circ} \mathrm{C}$ teve uma melhor inibição contra $\mathrm{o}$ Staphylococcus aureus $(52,6 \pm 2,1 \%)$, e a extração a $60^{\circ} \mathrm{C}$ comprovou-se mais eficaz para a inibição da Escherichia coli $(74,7 \pm 3,6 \%)$. 
Recebido em:

$27 / 08 / 2018$

Aceito em:

$12 / 11 / 2018$

Palavras-chave: Antibacteriano. Cogumelo ostra. Cogumelo pantorra.

DOI: $10.5965 / 24473650412018089$

\section{INTRODUÇÃO}

A globalização trouxe consigo um novo panorama do ponto de vista alimentício, em decorrência da necessidade de produtos industrializados e processos tecnológicos a fím de se aumentar a vida útil dos alimentos. Com isso um dos maiores problemas enfrentados pela indústria se refere a preservação destes produtos, uma vez que, segundo dados da Organização Mundial da Saúde, 20\% dos alimentos são perdidos devido a deterioração por micro-organismos (OS CONSERVANTES..., [2018]). Sendo assim, a necessidade de redução e/ou eliminação de micro-organismos deteriorantes e patógenos, a fim de se obter um alimento seguro e de qualidade, se torna uma necessidade (SILVA, 2007).

As barreiras mais usadas na conservação de alimentos geralmente são atividade de água, $\mathrm{pH}$, temperatura e conservantes, sabe-se que medidas combinadas aumentam a potencialidade da estabilidade microbiológica, qualidade sensorial, nutricional e econômica do alimento (SILVA, 2007). Os conservantes são usados a fim de produzir alimentos mais seguros para os consumidores, impedindo a ação de micro-organismos, prolongando assim a vida útil dos alimentos e evitando alterações no seu sabor e aparência (ALBUQUERQUE et al., 2012).

Juntamente com a produção exorbitante de produtos industrializados e utilização de aditivos químicos, teve-se o aumento do interesse por produtos naturais, em resposta a potencialidade de efeitos tóxicos destes produtos ao consumidor a longo prazo (NUNES, 2013). Antimicrobianos naturais são alternativas viáveis na substituição desses conservantes (GERHARDT et al., 2012), pois estudos relatam a potencialidade do efeito inibidor de condimentos e conservantes naturais no desenvolvimento de micro-organismos deteriorantes e patogênicos, veiculados a alimentos (PEREIRA, 2006).

A extração de conservantes naturais pode ser realizado a partir de fungos comestíveis, uma vez que, são facilmente encontrados no meio ambiente e apresentam uma potencialidade terapêutica e antimicrobiana consideráveis (DIAS et al., 2003). Estes fungos são divididos em três classes principais, dentre elas os Basidiomycotas que tem por seu principal representante fungos macroscópicos, os cogumelos, os quais são empregados na alimentação humana conhecido desde a antiguidade, apresentando-se como uma excelente fonte nutricional de sabor agradável (ZDRADEK, 2001).

O gênero Pleurotus pertence à ordem Agaricales e à família Agaricaceae, e dentro desse gênero podemos citar o Pleurotus sajor-caju (BORBA, 2013) que é um cogumelo de origem asiática, rico em vitaminas e aminoácidos conhecido comercialmente como cogumelo Ostra. $\mathrm{O}$ gênero Morchella pertence a ordem Pezizales (CORTEZ et al., 2004) e a família Morchellaceae, e como cogumelo comestível pertencente a este gênero podemos destacar o Morchella esculenta, de origem Francesa, rico em fibras e proteínas e conhecido comercialmente como Pantorra.

A capacidade de desenvolvimento do fungo está associada às condições de cultivo, como meio de cultura, acidez e temperatura que segundo Akinyele (2005) é um dos principais fatores ambientais que influência o crescimento de micro-organismos. $O$ cogumelo através de seu 
Recebido em:

$27 / 08 / 2018$

Aceito em:

$12 / 11 / 2018$

metabolismo fúngico metaboliza compostos para obtenção de nutrientes, estes compostos muitas vezes são bioativos, podendo, por exemplo, apresentar ação antimicrobiana frente a microorganismos patogênicos (AKINYELE, 2005).

Segundo o Ministério da Saúde (2018), os surtos de doenças transmitidas por alimentos notificados no Brasil entre os anos de 2000 à 2017, são decorrentes principalmente por bactérias, as quais são responsáveis por $92,2 \%$ destes. Sendo assim, diante da necessidade de conservantes eficazes sem que causem danos à saúde do consumidor, este trabalho objetivou a extração sob diferentes temperaturas de antimicrobianos do micélio de cogumelos Pleurotus sajor-caju e Morchella esculenta, ambos cultivados por fermentação em estado sólido, e a avaliação da sua ação frente aos micro-organismos Staphylococcus aureus e Escherichia coli.

\section{METODOLOGIA}

\subsection{Obtenção do extrato}

A extração do composto antimicrobiano deu-se a partir do micélio cultivado em placas de Petri contendo Ágar Batata Dextrose, por fermentação em estado sólido, cultivadas em estufa com circulação de ar a $25^{\circ} \mathrm{C}$. Após o desenvolvimento radial das hifas dos cogumelos estudados, o extrato foi obtido por raspagem do micélio com solvente e então submetido a agitação em mesa agitadora e agitador orbital na temperatura de 25 e $60^{\circ} \mathrm{C}$ respectivamente, sob agitação de 100 rpm por $1 \mathrm{~h}$, na sequência os extratos foram filtrados com membrana de $0,45 \mu \mathrm{m}$.

\subsection{Atividade antibacteriana}

A atividade antimicrobiana foi avaliada por meio do método de microplacas baseando-se na metodologia encontrada na norma NCCLS (2003). As microplacas utilizadas continham 6 poços cada um com $400 \mu \mathrm{L}$ de capacidade. Cada poço foi inoculado com caldo Mueller Hinton, extrato dos cogumelos $\left(25^{\circ} \mathrm{C}\right.$ e $\left.60^{\circ} \mathrm{C}\right)$ e micro-organismo Gram-positivo (Staphylococcus aureus) ou Gram-negativo (Escherichia coli), analisados separadamente.

Os resultados foram obtidos a partir da comparação do crescimento microbiano da amostra controle (sem adição de extrato) com os extratos obtidos. As leituras foram realizadas em leitora de microplacas que se dá através de leitura da absorbância em um comprimento de onda de 630 $\mathrm{nm}$ dos poços contendo as amostras logo depois de inoculadas e 16 horas após, de acordo com a NCCLS (2003).

\section{RESULTADOS E DISCUSSÃO}

Com as análises realizadas podemos observar a atividade antibacteriana dos cogumelos Pleurotus sajor-caju e Morchella esculenta através da Figura 1, onde pode-se ressaltar a inibição do desenvolvimento dos micro-organismos testados, assim como a diferença de inibição entre os cogumelos bem como entre as temperaturas utilizadas. 
Recebido em:

$27 / 08 / 2018$

Aceito em:

$12 / 11 / 2018$

Figura 1 - Atividade antibacteriana em porcentagem dos cogumelos comestíveis Pleurotus sajor-caju e Morchella esculenta em diferentes temperaturas frente à diferentes micro-organismos

(a) Escherichia coli

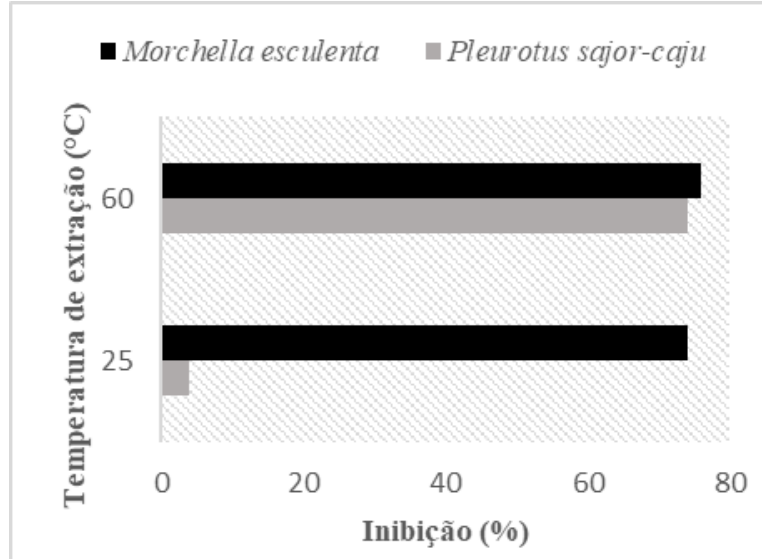

(b) Staphylococcus aureus

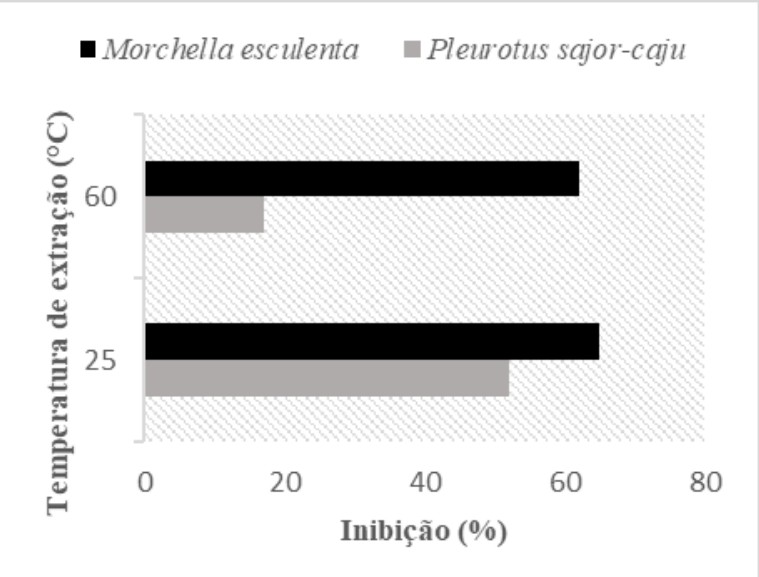

Fonte: elaborado pelos autores (2018)

Diante do exposto, nota-se que o extrato de Morchella esculenta assim como o de Pleurotus sajor-caju apresentaram de maneira geral atividade antibacteriana sob os microorganismos utilizados (Gram-positivo e Gram-negativo). Estes achados diferem do encontrado por Ghenov (2014), onde analisou a atividade antimicrobiana de extratos de Pleurotus ostreatus e Lentinula edodes e observou um halo de $11,0 \mathrm{~mm}$ para o extrato de Pleurotus ostreatus frente a Escherichia coli sem observar inibição contra Staphylococcus aureus. Assim como quando testado o extrato de ambos os cogumelos agindo em sinergismo, foi observado um halo de 4,5 $\mathrm{mm}$, apenas frente o mesmo micro-organismo Gram-negativo testado, constatando que o composto extraído dos fungos não apresentou atividade inibitória contra Gram-positivo. Negri (2012) também não obteve resultados positivos contra o desenvolvimento do micro-organismo Gram-positivo testado, constatando apenas atividade contra o grupo de Gram-negativos analisados quando testado o extrato de Agaricus blazei.

Contata-se ainda que não houve diferença significante na extração do composto antibacteriano de Morchella esculenta diante das temperaturas de extração, frente a microbiota utilizada, o que pode ser explicado possivelmente pela extração de apenas um composto, o qual possui ação contra bactérias de diferentes estruturas celulares e que mantem-se estável nas temperaturas estudadas, conforme Piovesan (2016) que também não obteve diferença nas temperaturas de extração de alguns compostos bioativos de mirtilo.

$\mathrm{O}$ extrato de Pleurotus sajor-caju apresentou um aumento de inibição na extração a $60^{\circ} \mathrm{C}$ de $4,2 \%$ para $74,7 \%$ sob a Escherichia coli, conforme Carvalho et al. (2003), esse comportamento pode ser explicado devido ao possível aumento da solubilidade do composto frente ao solvente, ocasionado pelo aumento da temperatura. Porém Barbosa et al. (2016), obtiveram um decréscimo na concentração de compostos fenólicos e da atividade antioxidante do extrato de milho de grãos pretos quando aumentou-se a temperatura de extração, sugerindo assim a possibilidade de que a temperatura de $60^{\circ} \mathrm{C}$ extraiu um composto que favorece a inibição de organismos Gram-negativos e a temperatura de $25^{\circ} \mathrm{C}$ se mostrou eficaz contra organismos Gram- 
Recebido em:

$27 / 08 / 2018$

Aceito em:

$12 / 11 / 2018$

positivos, havendo desta forma a possibilidade de mais de um composto de origem antibacteriana.

Conforme Carbonero et al. (2003), alguns polissacarídeos presentes em cogumelos, em específico as $\beta$-glucanas, as quais se fazem presentes no cogumelo Pleurotus sajor-caju, tem a capacidade de atuar como imunomoduladores e antimutagêncicos nos sistemas biológicos, podendo ser em decorrência da possível extração deste polissacarídeo a eficiência de inibição do extrato analisado.

É provável que tenham sido extraídos compostos antimicrobianos diferentes dos gêneros de cogumelos testados tendo em vista que foram cultivadas sob as mesmas condições e apresentaram diferentes porcentagens de inibição. O gênero Morchella se mostrou muito eficiente sob os dois patógenos testados, enquanto que o gênero Pleurotus também com resultados positivos de inibição teve comportamento diferente quanto as temperaturas de extração.

\section{CONSIDERAÇÕES FINAIS}

No presente trabalho foi possível comprovar que no extrato dos cogumelos testados existe compostos antimicrobianos que inibem o crescimento de micro-organismos patógenos como Escherichia coli e Staphylococcus aureus. Algumas considerações devem ser avaliadas quando usado extrato do Pleurotus sajor-caju, contudo deve ser analisado o patógeno de interesse, pois foi evidenciado que a extração feita do micélio do fungo a temperatura de $25^{\circ} \mathrm{C}$ teve uma melhor inibição contra o Staphylococcus aureus $(52,6 \pm 2,1 \%)$, e a extração a $60^{\circ} \mathrm{C}$ comprovou-se mais eficaz para a inibição da Escherichia coli $(74,7 \pm 3,6 \%)$. Quando pretende-se a utilização do extrato de Morchella esculenta como agente inibidor, o mesmo pode ser usado tanto para Grampositivos como para Gram-negativos pois se mostrou eficiente sob os dois micro-organismos testados indiferente da temperatura de extração, obtendo as porcentagens de 74,5 $\pm 3,7 \%$ na extração a $25^{\circ} \mathrm{C}$ e $76,3 \pm 2,5 \%$ quando submetido a extração a $60^{\circ} \mathrm{C}$ para Escherichia coli, e 65,8 $\pm 3,9 \%$ na extração a $25^{\circ} \mathrm{C}$ e $62,4 \pm 3,2 \%$ a $60^{\circ} \mathrm{C}$ para Staphylococcus aureus.

\section{AGRADECIMENTOS}

A Fundação de Amparo à Pesquisa do Estado do Rio Grande do Sul (FAPERGS), ao CNPq Processos 310385/2013-0 e 453878/2014-8 pelo apoio financeiro e incentivo a pesquisa e ao Laboratório de Microbiologia e Toxicologia de Alimentos da Universidade Federal do Pampa - campus Bagé, pelo espaço físico.

\section{REFERÊNCIAS}

AKINYELE, B. J.; ADETUYI, F. C. Effect of agrowastes, $\mathrm{pH}$ and temperature variation on the growth of Volva- riella volvacea. African Journal of Biotechnology, v. 4, n. 12, p. 1390-1395, 2005. 
ALBUQUERQUE, F. G. et al. Educação alimentar: uma proposta de redução do consumo de aditivos alimentares. Química Nova na Escola, v. 34, p. 51-57, 2012.

BARBOSA, N. A. et al. Influência da temperatura e do solvente na obtenção de extrato de milho de grãos pretos. XXXI Congresso Nacional de Milho e Sorgo, 2016.

BORBA, C. M. Extração de antimicrobianos de cogumelos dos gêneros Agaricus e Lentinula, cultivados em rejeitos agroindustriais por fermentação em estado sólido. Trabalho de Conclusão de Curso (Engenharia de Alimentos), Universidade Federal do Pampa, Bagé, 2013.

CARBONERO, E. R. et al. Análise química da $\beta$-glucana $(1 \rightarrow 3)(1 \rightarrow 6)$ isolada do corpo de frutificação do cogumelo comestível Pleurotus sajor-caju. 33 $^{\text {a }}$ Reunião Anual da Sociedade Brasileira de Química, 2003.

CARVALHO JUNIOR, R. N. et al. Determination of the global yield isotherms for the system rosemary (Rosimarinus officinalis) + CO2. International Symposium on Supercritical Fluids, p. 129-134, 2003.

CORTEZ, V. G. et al. Morchella Esculenta (Ascomycota): uma rara espécie encontrada em Santa Maria, Rio Grande Do Sul, Brasil. Biociências, v. 12, n. 1, p. 51-53, 2004.

DIAS, E. S. et al. Cultivo do cogumelo Pleurotus sajor-caju em diferentes resíduos agroindustriais. 2003. Ciênc. Agrotec., Lavrs. v. 27, n. 6, p. 1363-1369, 2003.

GERHARDT, C. et al. Aproveitamento da casca de citros na perspectiva de alimentos: prospecção da atividade antibacteriana. Food Technology. p. 11-17, 2012.

GHENOV, F. Avaliação in vitro das atividades antioxidante e antimicrobiana de extratos hidroalcoólicos de chá preto (Camellia sinensis) e dos cogumelos Shiitake (Pleurotus ostreatus) e Shimeji (Lentinula edodes). Trabalho de conclusão de curso (Graduação em química), Universidade Tecnológica Federal do Paraná, 2014.

MINISTÉRIO DA SAÚDE. Secretaria de Vigilância em Saúde. Surtos de Doenças Transmitidas por Alimentos no Brasil. 2018. Disponível em:

<http://portalarquivos2.saude.gov.br/images/pdf/2018/janeiro/17/Apresentacao-Surtos-DTA2018.pdf>. Acesso em: 14 ago. 2018.

NEGRI, K. M. S. Estudo comparativo de diferentes cogumelos comestíveis comercializados no Brasil: ação contra radicais livres, espécies reativas e atividade antibacteriana. Dissertação (Mestrado em Biotecnologia), Universidade Estadual Paulista "Júlio de Mesquita Filho", 2012. 
NUNES, M. A. S. Estudo de alternativas naturais a aditivos utilizados em produtos cárneos à base de aves na Empresa X. Dissertação (Mestrado em Tecnologia e Segurança Alimentar), Universidade de Nova Lisboa, 2013.

OS CONSERVANTES mais utilizados em alimentos. Aditivos \& Ingredientes. Disponível em: <http://aditivosingredientes.com.br/upload_arquivos/201601/2016010485708001453470366.pdf $\geq$. Acesso em: 14 ago. 2018.

PEREIRA, M. C. Inibição do desenvolvimento fúngico através da utilização de óleos essenciais de condimentos. Ciência e Agrotecnologia, v. 30, p. 731-738, 2006.

PIOVESAN, N. Influência de diferentes parâmetros em métodos de extração de compostos bioativos de mirtilo (Vaccinium ashei Reade) e atividade antioxidante e antimicrobiana. Tese (Doutorado em Ciência e Tecnologia de Alimentos), Universidade Federal de Santa Maria, 2016.

SILVA, J. P. L. Avaliação da ação de antimicrobianos naturais no controle de Salmonella Enteritidis em salada de legumes com maionese. 2007, 90 p., Tese (Doutorado em Ciência de Alimentos), Universidade de São Paulo, 2007.

ZDRADEK, C. P. Otimização do crescimento dos fungos comestíveis $\boldsymbol{P}$. ostreatus e $\boldsymbol{P}$. sajor caju utilizando resíduos agroindustriais. 139 p. Tese de Mestrado. Universidade Federal do Rio Grande, Rio Grande, 2001. 